\title{
ETUDE DENDROMETRIQUE DE Pterocarpus erinaceus POIR. DES FORMATIONS NATURELLES DE LA ZONE SOUDANIENNE AU BENIN
}

\author{
R. L. GLELE KAKAÏ', B. SINSIN ${ }^{1}$ et R. PALM ${ }^{2}$ \\ ${ }^{1}$ Laboratoire d'Ecologie Appliquée, Faculté des Sciences Agronomiques, Université d'Abomey-Calavi, 01 BP 526, \\ Cotonou (Bénin). E-mail : gleleromain@yahoo.fr \\ 2Unité de Statistique, Informatique et Mathématique appliquées, Faculté Universitaire des Sciences Agronomiques \\ de Gembloux (FUSAGx), Avenue de la Faculté d'Agronomie 8, B-5030, Gembloux (Belgique). \\ E-mail : palm.r@fsagx.ac.be
}

\begin{abstract}
RESUME
Pterocarpus erinaceus est une Légumineuse arborescente des forêts claires et savanes arborées d'Afrique, croissant en peuplements naturels dans les zones soudaniennes au Bénin. Un inventaire des arbres dans ces formations naturelles à dominance de $P$. erinaceus a été effectué afin de décrire leur structure, sur la base des paramètres dendrométriques comme la hauteur, le diamètre, la surface terrière, le facteur d'écorce, etc. Les mesures du diamètre et de la hauteur des arbres ont été réalisées dans 400 placettes circulaires de 15 mètres de rayon, situées dans les forêts claires et savanes arborées des forêts classées de l'Ouémé Supérieur et de Wari-Maro. Les résultats obtenus permettent de noter que la densité du peuplement est plus élevée dans les formations de savane (169,4 arbres/ha) alors que la hauteur moyenne et le diamètre moyen de tous les arbres et spécifiquement de $P$. erinaceus présentent des valeurs significativement plus élevées en forêt. Par ailleurs, les deux forêts étudiées (Ouémé Supérieur et WariMaro) présentent des valeurs similaires pour les paramètres dendrométriques calculés. La structure en diamètre de $P$. erinaceus révèle une relative stabilité du peuplement avec une abondance des individus de diamètre moyen et une rareté des individus de faibles ou gros diamètres. L'ensemble du peuplement présente une structure en " $\mathrm{i}$ », caractéristique d'une nature inéquienne et multispécifique. La hauteur de $P$. erinaceus peut être exprimée en fonction du diamètre à $1,30 \mathrm{~m}$ du sol, dans les peuplements de savane et de forêt par des équations logarithmiques avec des coefficients de détermination de $37 \%$ et $16 \%$ respectivement en savane arborée et forêt claire.
\end{abstract}

Mots-Clés : Pterocarpus erinaceus, formations naturelles, paramètres dendrométriques, zone soudanienne, Bénin.

\section{ABSTRACT}

DENDROMETRIC STUDY OF PterocarpUS erinaceuS POIR IN THE NATURAL STANDS OF SUDANIAN ZONE OF BENIN

Pterocarpus erinaceus is an arborescent deciduous legume tree of African savannas and dry forests growing in natural stands in the sudanian zone in Benin. An inventory of trees in the P. erinaceus dominated communities was carried out in order to describe their structure in tree-savannah and woodland, on the basis of some dendrometric parameters such as the diameter, the height, the basal area, the bark factor etc. The measurements have been done in 400 circular plots of $15 \mathrm{~m}$ of radius, in Ouémé Supérieur and Wari-Maro forest reserves. The results showed that the tree-density of the stands was higher in tree-savannas than in the woodlands whereas the mean height and the mean diameter of $\mathrm{P}$. erinaceus trees and of the whole stands obtained their highest values in woodlands. Furthermore, the two targeted forest reserves (Ouémé supérieur and Wari-Maro) have similar values for the dendrometric parameters. The diameter structure of $\mathrm{P}$. erinaceus revealed the abundance of trees with mean diameter whereas trees with small or high diameter were scarce. The overall stand has an «i-shaped» structure, showing an uneven-aged and mixed species nature of the stands. Moreover, the height-diameter relationship of $\mathrm{P}$. erinaceus was logarithmic with a $R$-square value of $37 \%$ and $16 \%$ in tree-savanna and woodland, respectively.

Keywords : Pterocarpus erinaceus, natural stands, dendrometric parameters, sudanian zone, Benin. 


\section{INTRODUCTION}

Les produits forestiers constituent des ressources importantes pour toutes les nations en raison de leur rôle notable dans les économies des pays. Les flux mondiaux des produits du bois ont mobilisé, en 2003, une somme de 117,66 milliards de dollars US (Anonyme, 2006). Malheureusement, du fait de plusieurs facteurs dont la rareté de plans d'aménagement forestier adéquats, la pression démographique dans le monde et particulièrement en Afrique et les pratiques anthropiques (agriculture, chasse, élevage, transhumance, exploitation abusive), les produits forestiers sont en baisse constante.

Au Bénin, pays à faible potentiel forestier (Anonyme, 1981), on note une forte pression anthropique sur les maigres ressources forestières disponibles. Les trois essences forestières autochtones faisant objet de demande préférentielle en bois d'œuvre et de service sont Khaya senegalensis (Desr.), Afzelia africana (Sm.) et Pterocarpus erinaceus Poir. $K$. senegalensis et $A$. africana, jadis bien représentées dans les forêts, sont devenues rares (Sokpon, 2000, Sinsin et al., 2004). La troisième essence, à savoir $P$. erinaceus (le vêne), disponible encore en peuplements natuels dans les forêts claires et savanes arborées, entre $8^{\circ}$ et $10^{\circ} \mathrm{N}$ de latitude, est exploitée de façon abusive comme bois d'œuvre et de service.

Cette dernière espèce forestière grégaire subit au Bénin des pressions de plus en plus fortes de déboisement par écrémage. Cette situation est aggravée par le fait que l'espèce n'existe jusqu'aujourd'hui qu'en peuplements naturels et n'a pas encore fait l'objet d'étude d'aménagement, ni d'étude de régénération au Bénin. II est donc important de connaître l'état actuel des peuplements de $P$. erinaceus à travers une étude structurale, base d'un aménagement adéquat.

Le but de la présente étude est de décrire les caractéristiques dendrométriques des populations de $P$. erinaceus pour un aménagement futur. Les objectifs spécifiques sont d'une part de déterminer les caractéristiques dendrométriques des peuplements naturels à dominance de $P$. erinaceus dans les forêts classées de Wari-Maro et de l'Ouémé Supérieur et d'autre part, de décrire la structure en fonction du diamètre et de la hauteur totale des arbres des peuplements de l'espèce en forêt claire et en savane arborée de la zone soudanienne au Bénin. Les hypothèses testées lors de l'étude étaient relatives à l'égalité des moyennes des valeurs des paramètres dendrométriques, entre savanes et forêts d'une part et entre les forêts classées de Wari-Maro et de l'Ouémé Supérieur d'autre part.

\section{MATERIEL ET METHODES}

\section{MILIEUD'ETUDE}

Le milieu d'étude est constitué des formations naturelles à dominance de $P$. erinaceus de la zone soudanienne du Bénin. Elles sont situées précisément dans les forêts classées de WariMaro et de l'Ouémé Supérieur. La forêt classée de Wari-Maro, avec une superficie estimée à 120686 ha, est située au Centre du Bénin, entre les latitudes $8^{\circ} 80$ et $9^{\circ} 10 \mathrm{~N}$ et les longitudes $1^{\circ} 55$ et $2^{\circ} 25 \mathrm{E}$. C'est la zone de transition soudano-guinéenne définie par White (1983) comme celle des forêts claires à Isoberlinia spp. Elle est limitée au Nord par la forêt classée de l'Ouémé Supérieur (figure 1), au Sud par la forêt classée des Monts-Kouffé, à l'Est par l'axe national Tchaourou-Bétérou et à l'Ouest par la piste Wari-Maro-Igbèrè. La forêt classée de l'Ouémé Supérieur est située entre les latitudes $9^{\circ} 11$ et $9^{\circ} 47 \mathrm{~N}$ et longitudes $1^{\circ} 58$ et $2^{\circ} 28 \mathrm{E}$. Sa superficie est estimée à 193400 ha. Les deux forêts classées sont sous l'influence du climat tropical de type soudanien humide caractérisé par deux saisons de durées presque égales : la saison sèche marquée s'étend de novembre à mars et peut être divisée en deux saisons thermiques: une saison froide de décembreà février due au passage de l'harmattan et une saison chaude de mars à avril. La saison pluvieuse s'établit d'avril à octobre avec $1247 \mathrm{~mm}$ de pluviométrie moyenne par an. 


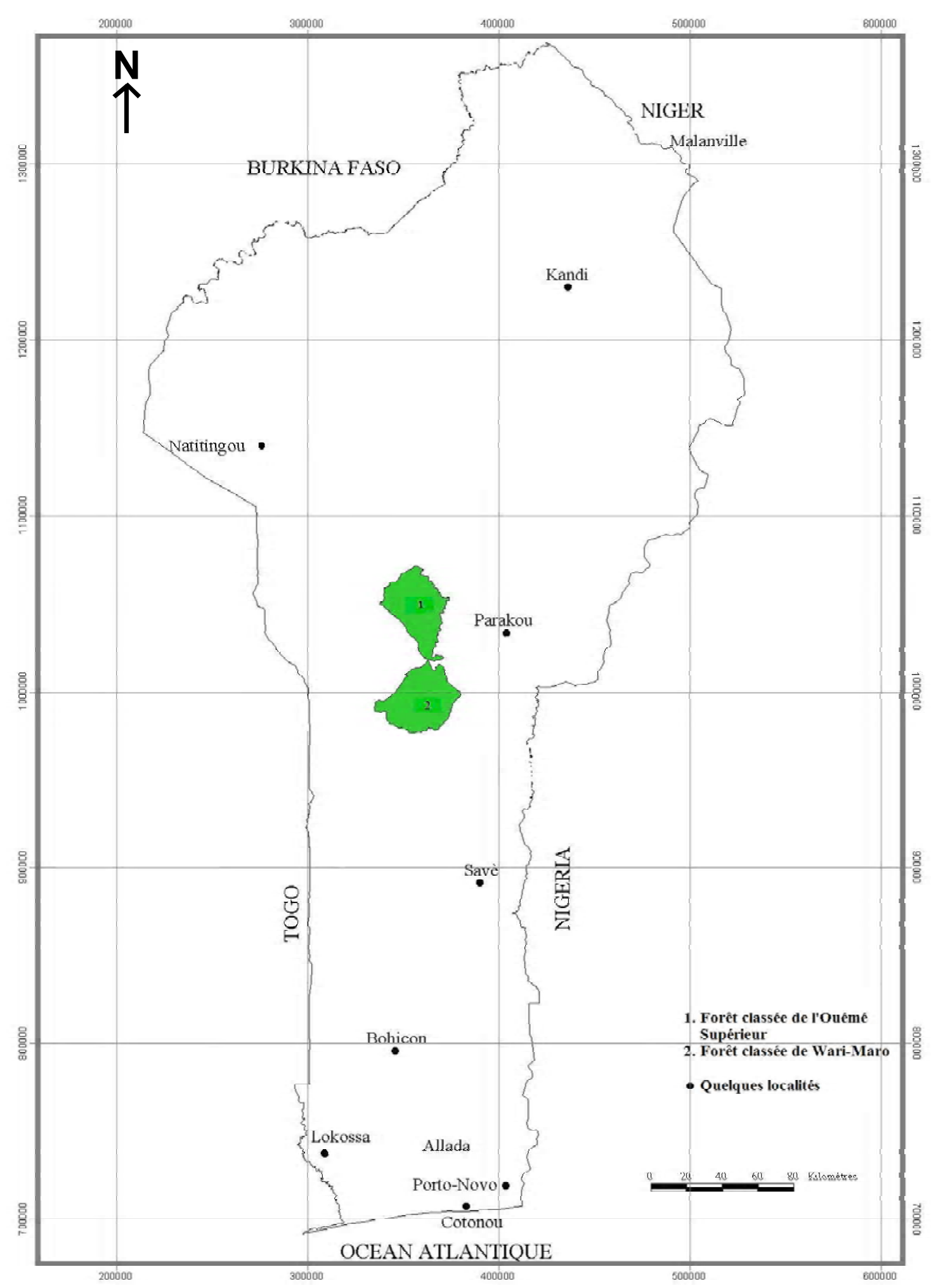

Figure 1 : Localisation des forêts classées de l'Ouémé Supérieur et de Wari-Maro. Location of Ouémé Supérieur and Wari-Maro forest reserves. 


\section{MATERIEL VEGETAL}

Le matériel végétal considéré dans la présente étude est constitué des peuplements naturels à dominance de $P$. erinaceus installés à divers endroits dans les forêts classées de l'Ouémé Supérieur et de Wari-Maro.

$P$. erinaceus est une Légumineuse de la sousfamille des Papilionoideae, utilisée en ébénisterie pour la qualité de son bois et en nutrition des ruminants en saison sèche du fait de la richesse de ses feuilles en protéine (Bonkoungou et al., 1999). C'est un arbre souvent rencontré en Afrique de l'Ouest et du Centre depuis le Sénégal jusqu'à la République Centrafricaine. II est présent jusqu'à la latitude $14^{\circ} \mathrm{N}$, en populations de petite taille. Par ailleurs, $P$. erinaceus n'a pas encore fait l'objet d'introduction dans une région hors de son aire géographique (Bonkoungou et al., 1999).

\section{METHODE DE COLLECTE DES DONNEES}

Les données ont été collectées dans des unités d'observation des deux forêts classées. Le repérage des peuplements naturels à dominance de $P$. erinaceus est fait en tenant compte de la description des groupements végétaux (Yayi,
1998). Les unités d'observation sont délimitées suivant un échantillonnage simple non probabiliste. Les unités du premier degré sont des parcelles de forme carrée d'un hectare de superficie, identifiées dans les peuplements et distantes l'une de l'autre de $200 \mathrm{~m}$. Dans chaque parcelle, sont ensuite installées quatre placettes circulaires de $15 \mathrm{~m}$ de rayon aux sommets de la parcelle (figure 2).

Soixante parcelles ont été mises en place dans les formations à dominance de $P$. erinaceus de la forêt classée de l'Ouémé Supérieur et 40 dans celles de la forêt classée de Wari-Maro. Les données ont été collectées dans 400 placettes de 7 ares environ (soit sur une superficie totale de 28 ha) réparties entre 319 placettes en savane arborée et 81 placettes situées en forêt claire. Dans chaque placette, les mesures ont été réalisées sur les arbres de toutes espèces ayant un diamètre à hauteur d'homme ( $\mathrm{dbh}$ ) supérieur à $10 \mathrm{~cm}$ à $1,30 \mathrm{~m}$ du sol. II s'agit du diamètre à $1,30 \mathrm{~m}$ au-dessus du sol mesuré au compas forestier, la hauteur totale, mesurée avec le clinomètre uniquement sur les individus de $P$. erinaceus et l'épaisseur de l'écorce de $P$. erinaceus, évaluée en faisant une petite entaille à l'aide d'une machette et un décimètre.

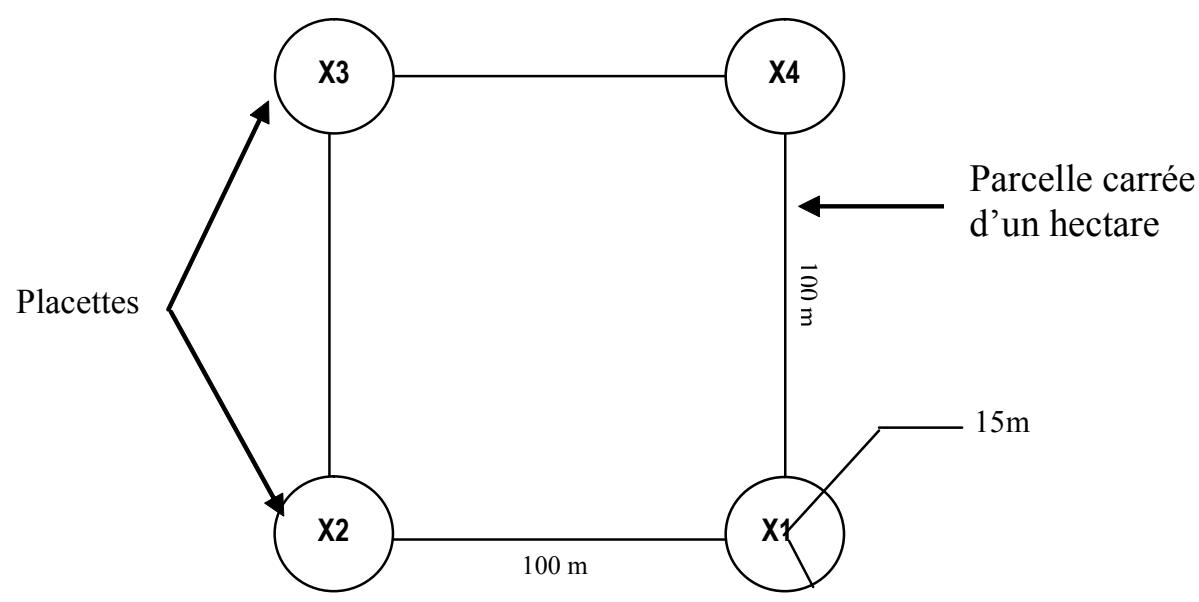

Figure 2 : Représentation des placettes au sein d'une parcelle d'un hectare. Location of the plots in a land parcel of one hectare.

\section{ANALYSE DES DONNEES}

\section{Paramètres dendrométriques des peuplements}

Six paramètres dendrométriques ont été retenus pour l'étude : la densité, la surface terrière, le diamètre, la hauteur moyenne de Lorey, le facteur d'écorce et la contribution en surface terrière.

La densité du peuplement $(\mathrm{N})$ est le nombre moyen d'arbres sur pied ramené à l'hectare, calculée par la formule :

$$
\mathrm{N}=\frac{\mathrm{n}}{\mathrm{s}}
$$


avec $\mathrm{n}$ : nombre d'arbres de la placette, et $\mathrm{s}$ : surface de la placette (en ha); s=0,0225 ha.

La surface terrière $(G)$ en $\mathrm{m}^{2} /$ ha, est la somme des sections transversales à 1,30 m de hauteur du sol, de tous les arbres de la placette:

$$
\mathrm{G}=\frac{\pi}{4 s} \sum_{\mathrm{i}=1}^{\mathrm{n}} \mathrm{d}_{\mathrm{i}}^{2}
$$

avec $\mathrm{d}_{\mathrm{i}}$ : diamètre (en $\mathrm{cm}$ ) de l'arbre i de la placette et $\mathrm{s}$ : superficie de la placette.

Le diamètre de l'arbre de surface terrière moyenne (D) en cm est obtenu par la relation :

$$
D=\sqrt{\frac{1}{n} \sum_{i=1}^{n} d_{i}^{2}}
$$

avec $\mathrm{n}$ : nombre d'arbres de la placette; $\mathrm{d}_{\mathrm{i}}$ : diamètre de l'arbre i (en $\mathrm{cm}$ ).

La hauteur moyenne de Lorey $(\mathrm{H})$ en $\mathrm{cm}$, est la hauteur moyenne des arbres, pondérée par leur surface terrière (Philip, 2002):

$$
H=\left(\sum_{i=1}^{n} g_{i} h_{i}\right) /\left(\sum_{i=1}^{n} g_{i}\right) ; g_{i}=\frac{\pi}{4} d_{i}^{2}
$$

avec $g_{i}$ : surface terrière (en $\left.\mathrm{m}^{2} / \mathrm{ha}\right) ; \mathrm{h}_{\mathrm{i}}$ : hauteur de l'arbre i.

Les quatre paramètres dendrométriques définis ci-dessus sont calculés d'une part, pour l'ensemble des arbres de chaque placette inventoriée et, d'autre part, de manière spécifique pour $P$. erinaceus.

Le facteur d'écorce $(\mathrm{k})$ est calculé pour $P$. erinaceus en vue d'apprécier l'importance de la proportion de bois utile dans le volume en bois. Il est défini de la manière suivante:

$k=\left(\sum_{i=1}^{n^{\prime}} d_{u i}\right) /\left(\sum_{i=1}^{n^{\prime}} d p_{i}\right) ; d_{u i}=d p_{i}-2 e_{i}$

avec $\mathrm{e}_{\mathrm{i}}$ : épaisseur de l'écorce mesurée sur l'arbre ; $\mathrm{dp}_{\mathrm{i}}$ : diamètre sur écorce de l'arbre $\mathrm{i}$; n' : nombre de $P$. erinaceus dans la placette.
La contribution en surface terrière (Cs) en pourcent, est définie comme étant la part de $P$. erinaceus dans la surface terrière de l'ensemble des arbres de la placette :

$$
\mathrm{Cs}=100 \mathrm{Gp} / \mathrm{G}
$$

avec Gp : surface terrière des arbres de $P$. erinaceus ; $G$ : surface terrière de l'ensemble des arbres de la placette.

Les six paramètres dendrométriques définis cidessus sont calculés par placette. Ensuite, la moyenne est calculée pour les quatre placettes appartenant à une même parcelle d'un hectare. On dispose donc de six valeurs moyennes des paramètres dendrométriques pour chacune des 100 parcelles considérées dans les deux forêts classées. La moyenne et le coefficient de variation des six paramètres sont déterminés, d'une part, pour les formations naturelles de savane (80 parcelles) et de forêt (20 parcelles) et, d'autre part, pour chacune des deux forêts considérées (40 parcelles dans la forêt de WariMaro et 60 parcelles dans la forêt de l'Ouémé Supérieur). Le test $t$ de Student est ensuite effectué sur chacun des six paramètres afin de comparer les deux types de formation et les deux forêts considérées. Par ailleurs, l'indice de Blackman $\mathrm{I}_{\mathrm{B}}$, a été calculé pour caractériser la distribution spatiale des populations de $P$. erinaceus dans les formations de savane et de forêt. Cet indice est calculé à partir de la relation:

$$
\mathrm{I}_{\mathrm{B}}=\sigma_{\mathrm{N}}^{2} / \mu_{\mathrm{H}}
$$

$\operatorname{avec} \sigma_{\mathrm{N}}^{2}$ : variance des densités, $\mu_{\mathrm{N}}$ : densité moyerime des arbres des populations de $P$. erinaceus.

\section{Etablissement de la structure en diamètre des peuplements}

La structure en diamètre d'un peuplement décrit l'abondance relative des arbres par classe de diamètre. Pour établir cette structure, tous les arbres ont été regroupés en classes de diamètre de $5 \mathrm{~cm}$ d'amplitude et des histogrammes ont été construits. La distribution normale tronquée 
à gauche à $10 \mathrm{~cm}$ a été ajustée dans le cas de la structure en diamètre de $P$. erinaceus alors que la distribution exponentielle négative tronquée aussi à gauche à $10 \mathrm{~cm}$ est ajustée pour le peuplement global. Le seuil de troncature de $10 \mathrm{~cm}$ est lié au protocole d'inventaire forestier utilisé, qui ne prend en compte que les arbres d'au moins $10 \mathrm{~cm}$ de diamètre. Le test $x^{2}$ d'ajustement est ensuite effectué dans les deux cas pour vérifier l'adéquation des modèles.

\section{Etablissement de la relation hauteur- diamètre et caractérisation du profil de $P$. erinaceus.}

La relation entre la hauteur totale et le diamètre des arbres, encore appelée courbe des hauteurs (Rondeux, 1999), est un outil permettant de diminuer le coût et le temps de réalisation d'inventaires forestiers ultérieurs parce qu'elle permet d'avoir une idée de la hauteur d'un arbre, connaissant son diamètre. Cette relation est établie et représentée sous forme graphique pour chacune des deux formations. Les deux modèles suivants ont été testés :

$h=a+b \log d$ (Modèle de Hendricksen)

et $\operatorname{logh}=a+b$ logd (Modèle de Stoffels et Van Soest),

$\mathrm{h}$ : hauteur de l'arbre $(\mathrm{en} \mathrm{m}) ; \mathrm{d}$ : diamètre $(\mathrm{en} \mathrm{cm})$; log : logarithme népérien ; a : ordonnée a l'origine ; $b$ : pente logarithmique.

\section{RESULTATS}

\section{PARAMETRES DENDROMETRIQUES}

Les résultats obtenus pour les six paramètres dendrométriques (Tableau 1) montrent que la densité globale $(\mathrm{N})$ des arbres est significativement plus importante en savane (169 tiges/ha) qu'en forêt (147 tiges/ha).

Tableau 1 : Moyennes, coefficients de variation (cv) et valeurs de probabilité du test t relatif aux paramètres dendrométriques des peuplements de $P$. erinaceus en savane et en forêt.

Means, coefficients of variation (cv) and p-values of the $t$-tests of dendrometric parameters of $\mathrm{P}$. erinaceus stands in tree savannah and woodland.

\begin{tabular}{|c|c|c|c|c|c|}
\hline \multirow{2}{*}{ Paramètres } & \multicolumn{2}{|c|}{ Savane } & \multicolumn{2}{|c|}{ Forêt } & \multirow{2}{*}{ Prob. } \\
\hline & Moyenne & $\mathrm{CV}(\%)$ & Moyenne & CV (\%) & \\
\hline \multicolumn{6}{|c|}{ Peuplement global } \\
\hline $\begin{array}{l}\text { Densité des arbres, } \\
\text { N (tiges/ha) }\end{array}$ & 169,4 & 32,5 & 146,6 & 35,5 & 0,005 \\
\hline Diamètre, D (cm) & 27,91 & 20,3 & 32,39 & 21,1 & 0,000 \\
\hline $\begin{array}{l}\text { Surface terrière, } \\
G\left(\mathrm{~m}^{2} / \mathrm{ha}\right)\end{array}$ & 10,57 & 46,7 & 11,84 & 37,9 & 0,069 \\
\hline Hauteur (m) & 13,39 & 19,6 & 16,64 & 17,3 & 0,000 \\
\hline \multicolumn{6}{|c|}{ P. erinaceus } \\
\hline $\begin{array}{l}\text { Densité des arbres, } \\
\text { Np (tiges/ha) }\end{array}$ & 22,86 & 94,5 & 23,36 & 91,4 & 0,873 \\
\hline Diamètre, Dp (cm) & 36,91 & 31,6 & 40,86 & 29,2 & 0,048 \\
\hline $\begin{array}{l}\text { Surface terrière, } \\
\text { Gp }\left(\mathrm{m}^{2} / \mathrm{ha}\right)\end{array}$ & 2,54 & 110,0 & 3,60 & 75,3 & 0,269 \\
\hline Hauteur, Hp (m) & 13,44 & 24,3 & 16,28 & 21,1 & 0,000 \\
\hline Facteur d'écorce, k & 0,89 & 4,9 & 0,89 & 3,9 & 0,553 \\
\hline $\begin{array}{l}\text { Contribution en surface } \\
\text { terrière, Cs }(\%)\end{array}$ & 26,03 & 62,8 & 29,12 & 61,4 & 0,292 \\
\hline
\end{tabular}


Pour les autres paramètres, la probabilité liée au test $t$ est supérieure à 0,05 . Les valeurs moyennes globales de ces paramètres déterminées pour l'ensemble des deux formations sont de $10,8 \mathrm{~m}^{2} / \mathrm{ha} ; 23$ arbres/ha ; $3,2 \mathrm{~m}^{2} /$ ha $; 0,9$ et $26,7 \%$, respectivement pour la surface terrière globale, la densité, la surface terrière, le facteur d'écorce et la contribution spatiale de $P$. erinaceus. Dans les formations de savane, le diamètre moyen de l'ensemble des arbres est de $27,9 \mathrm{~cm}$ contre $36,9 \mathrm{~cm}$ pour $P$. erinaceus. En forêt, le diamètre moyen de l'ensemble des arbres est de $32,39 \mathrm{~cm}$ contre $40,86 \mathrm{~cm}$ pour $P$. erinaceus ; la probabilité liée au test $t$ (échantillons associés par paires) est inférieure à 0,001 dans les deux cas. Pour la hauteur totale moyenne, on ne note pas de différence significative entre $P$. erinaceus et les autres arbres.

Quant à la distribution spatiale des peuplements à dominance de $P$. erinaceus, l'indice de
Blackman est de 20,4 et 19,5, respectivement pour les peuplements de savane et de forêt.

Les valeurs moyennes globales des six paramètres dendrométriques par forêt classée sont présentées au tableau 2 . On y note que la densité moyenne et le diamètre moyen des arbres de Pterocarpus erinaceus sont estimés à 22,3 tiges/ha et $38,07 \mathrm{~cm}$ dans la forêt classée de l'Ouémé Supérieur et de 27,4 tiges/ha et $35,49 \mathrm{~cm}$ dans la forêt classée de Wari-Maro.

Les paramètres dendrométriques des autres essences de valeur retrouvées dans les peuplements à dominance de $P$. erinaceus en savane et en forêt sont résumés dans le tableau 3. On y note que la densité des principales essences varie de 0,04 à 22,86 tiges/ha en savane et de 0,18 à 23,36 tiges/ha en forêt , la surface terrière varie de $0,01 \mathrm{~m}^{2} /$ ha à $2,54 \mathrm{~m}^{2} / \mathrm{ha}$ en savane et de $0,001 \mathrm{~m}^{2} / \mathrm{ha}$ à $3,6 \mathrm{~m}^{2} /$ ha en forêt.

Tableau 2 : Moyennes, coefficients de variation (cv) et valeurs de probabilité du test t relatif aux paramètres dendrométriques des peuplements de $P$. erinaceus des forêts de Wari-Maro et de l'Ouémé Supérieur.

Means, coefficients of variation (cv) and p-values of the t-tests of dendrometric parameters of $\mathrm{P}$. erinaceus stands in Wari-Maro and Ouémé Supérieur forest reserves.

\begin{tabular}{|c|c|c|c|c|c|}
\hline \multirow{2}{*}{ Paramètres } & \multicolumn{2}{|c|}{ Ouémé supérieur } & \multicolumn{2}{|c|}{ Wari-Maro } & \multirow{2}{*}{ Prob. } \\
\hline & Moyenne & CV (\%) & Moyenne & CV (\%) & \\
\hline & \multicolumn{4}{|c|}{ Peuplement global } & \\
\hline $\begin{array}{l}\text { Densité des arbres, } \\
\mathrm{N} \text { (tiges/ha) }\end{array}$ & 164,0 & 34,5 & 169,8 & 27,1 & 0,498 \\
\hline Diamètre, D $(\mathrm{cm})$ & 28,75 & 22,1 & 29,27 & 16,9 & 0,576 \\
\hline $\begin{array}{l}\text { Surface terrière, } \\
G\left(\mathrm{~m}^{2} / \mathrm{ha}\right)\end{array}$ & 10,72 & 45,9 & 11,56 & 39,0 & 0,310 \\
\hline \multirow[t]{2}{*}{ Hauteur $(m)$} & 13,94 & 20,6 & 14,74 & 24,3 & 0,211 \\
\hline & \multicolumn{4}{|c|}{ P. erinaceus } & \\
\hline $\begin{array}{l}\text { Densité des arbres, } \\
\text { Np (tiges/ha) }\end{array}$ & 22,30 & 96,4 & 27,40 & 78,5 & 0,191 \\
\hline Diamètre, Dp (cm) & 38,07 & 30,5 & 35,49 & 35,6 & 0,293 \\
\hline $\begin{array}{l}\text { Surface terrière, } \\
\text { Gp }\left(\mathrm{m}^{2} / \mathrm{ha}\right)\end{array}$ & 2,59 & 108,9 & 2,80 & 96,1 & 0,673 \\
\hline Hauteur, $\mathrm{Hp}(\mathrm{m})$ & 14,09 & 23,6 & 13,53 & 32,8 & 0,507 \\
\hline $\mathrm{k}$ & 0,89 & 4,8 & 0,90 & 4,4 & 0,520 \\
\hline Cs (\%) & 21,72 & 84,3 & 22,63 & 79,6 & 0,776 \\
\hline
\end{tabular}


Tableau 3 : Paramètres dendrométriques des essences de valeur des peuplements à dominance de $P$. erinaceus en savane et en forêt.

Dendrometric parameters of the valuable forest species of $\mathrm{P}$. erinaceus stands in treesavannah and woodland.

\begin{tabular}{lcccccc}
\hline \multirow{1}{*}{ Espèces } & \multicolumn{5}{c}{ Savane } & Forêt \\
\cline { 2 - 7 } & $\begin{array}{c}\text { Densité, } \\
\mathrm{N} \text { (tiges/ha) }\end{array}$ & $\begin{array}{c}\text { Surface terrière, } \\
\mathrm{G}\left(\mathrm{m}^{2} / \mathrm{ha}\right)\end{array}$ & $\begin{array}{c}\text { Diamètre, } \\
\mathrm{D}(\mathrm{cm})\end{array}$ & $\begin{array}{c}\text { Densité, } \\
\mathrm{N}(\mathrm{tiges} / \mathrm{ha})\end{array}$ & $\begin{array}{c}\text { Surface terrière, } \\
\mathrm{G}\left(\mathrm{m}^{2} / \mathrm{ha}\right)\end{array}$ & $\begin{array}{c}\text { Diamètre, } \\
\mathrm{D}(\mathrm{cm})\end{array}$ \\
\hline $\begin{array}{l}\text { Afzelia africana } \\
\text { Anogeissus }\end{array}$ & 1,37 & 0,29 & 51,5 & 0,89 & 0,11 & 39,9 \\
leiocarpa & 3,27 & 0,36 & 37,6 & 11,20 & 1,14 & 35,9 \\
$\begin{array}{l}\text { Burkea africana } \\
\text { Combretum spp. }\end{array}$ & 10,31 & 0,50 & 24,8 & 8,71 & 0,39 & 24,0 \\
Daniella oliverii & 1,90 & 0,07 & 21,4 & 1,96 & 0,10 & 26,0 \\
Detarium & 4,29 & 0,45 & 36,4 & 3,73 & 0,72 & 49,4 \\
microcarpum & 3,01 & 0,09 & 19,9 & 0,18 & 0,00 & 11,2 \\
Isoberlinia spp & 21,90 & 2,18 & 35,6 & 21,16 & 2,40 & 38,0 \\
Khaya spp. & 0,04 & 0,01 & 45,9 & 0,36 & 0,02 & 27,1 \\
Lannea acida & 5,35 & 0,36 & 29,1 & 6,40 & 0,54 & 32,7 \\
Prosopis africana & 2,35 & 0,16 & 29,3 & 1,60 & 0,11 & 29,6 \\
P. erinaceus & 22,86 & 2,54 & 36,9 & 23,36 & 3,60 & 40,9 \\
\hline
\end{tabular}

\section{STRUCTURE DES FORMATIONS NATURELLES DE $P$. erinaceus}

\section{Structure en diamètre de l'ensemble des arbres}

Les répartitions en classes de grosseur de l'ensemble des arbres mesurés sont présentées pour les deux formations naturelles à la figure 3. II s'agit de distributions en «i». Les histogrammes montrent également, en surimposition, le résultat de l'ajustement d'une distribution exponentielle tronquée à gauche.

Bien que les écarts entre les fréquences théoriques et les fréquences observées soient statistiquement significatifs ( $p=0,03)$, on peut constater que les distributions observées ont bien l'allure générale d'une distribution exponentielle négative.
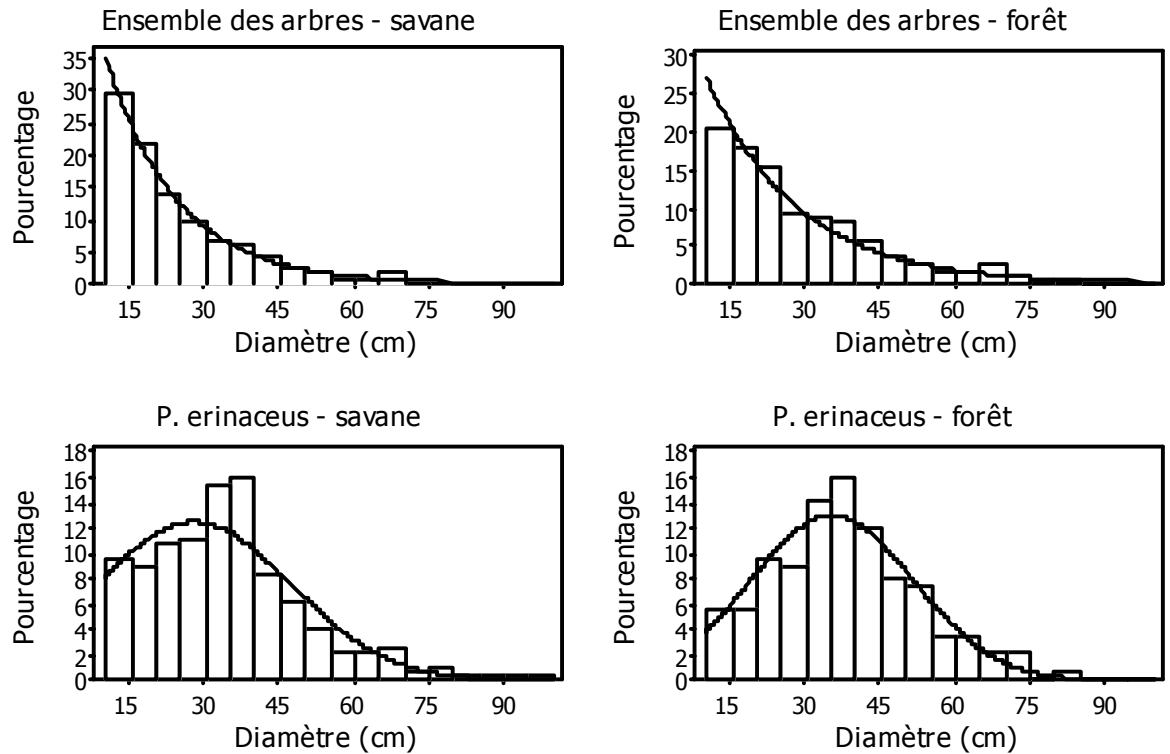

Figure 3 : Distributions par classe de diamètre de l'ensemble des arbres et de $P$. erinaceus en savane et en forêt.

Stem diameter structures of all trees and of $\mathrm{P}$. erinaceus in tree-savannah and in woodland. 


\section{Structure en diamètre de $P$. erinaceus}

La répartition en diamètre de $P$. erinaceus est présentée à la figure 3 avec, en surimpression, le résultat de l'ajustement d'une distribution normale tronquée. Les tests d'ajustement réalisés montrent que les écarts entre les fréquences observées et les fréquences estimées sont significatifs pour les savanes mais non significatifs pour les forêts.

\section{FORME DE L'ARBRE}

La forme de l'arbre est décrite d'une part, par la relation entre la hauteur et le diamètre à 1,30 m et d'autre part, par l'équation de défilement. Les relations hauteur-diamètre, qui permettent d'estimer au mieux la hauteur d'un arbre de $P$. erinaceus connaissant son diamètre, sont illustrées à la figure 4. Les équations obtenues sont :

Forêt :

$h=-1,5+4,21 \log d$ (Modèle de Hendricksen)

$R^{2}=16,2 \%$.

Savane :

$\operatorname{logh}=0,758+0,5 \log d$ (Modèle de Stoffels et Van Soest)

$\mathrm{R}^{2}=37,1 \%$.

On note que le diamètre explique respectivement $16 \%$ et $37 \%$ de la variabilité des hauteurs respectivement dans les peuplements de forêt et dans les peuplements de savane.

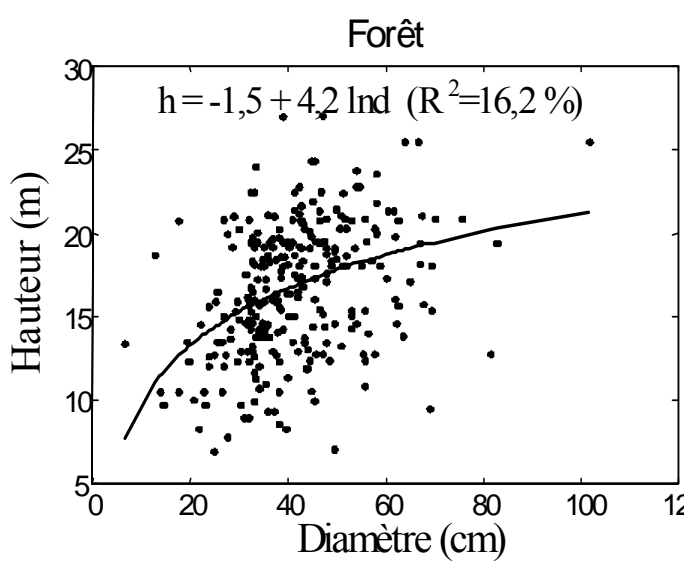

Figure 4 : Evolution de la hauteur des arbres de $P$. erinaceus en fonction du diamètre. Height-diameter curve of $\mathrm{P}$. erinaceus.

\section{DISCUSSION}

\section{PARAMETRES DENDROMETRIQUES DES PEUPLEMENTS}

L'étude dendrométrique des peuplements naturels de $P$. erinaceus a été faite dans les forêts classées de l'Ouémé Supérieur et de WariMaro à partir d'un inventaire forestier. Nous avons noté de cette étude que les valeurs de certains paramètres dendrométriques dépendent de la formation naturelle hébergeant les peuplements de $P$. erinaceus. Les peuplements sont plus denses en savane qu'en forêt alors que le diamètre et la hauteur des arbres du peuplement, ainsi que le diamètre et la hauteur de $P$. erinaceus présentent des valeurs plus élevées en forêt. Les peuplements de $P$. erinaceus des savanes soudaniennes du Bénin sont en effet composés, en quantité relativement importante, d'arbustes et d'arbres de petites tailles alors qu'en forêt claire, la compétition interspécifique fait qu'on rencontre moins d'arbres mais ceuxci sont de plus grande taille. Les valeurs moyennes de densité notées dans les peuplements de savane et de forêt $(169,4$ et 146,6 arbres/ha) sont nettement inférieures à celles obtenues précédemment par Yayi (1998) dans la même forêt (376 arbres/ha) et Sagbo (2000) dans les peuplements à dominance de Isoberlinia spp. (205 arbres/ha). Ces écarts peuvent être, en partie, dus aux méthodes d'inventaire utilisées et aussi au fait que les peuplements inventoriés ne sont pas exactement les mêmes. Ils peuvent également traduire, en partie, la forte pression anthropique exercée par les populations riveraines sur les essences forestières de valeur. 
La hauteur moyenne de $P$. erinaceus obtenue en savane $(13,4 \mathrm{~m})$ est supérieure à celle obtenue par Petit (2000) dans les savanes au Burkina-Faso (11 m). Notons que des arbres de $P$. erinaceus atteignant $35 \mathrm{~m}$ de hauteur sont signalés par von Maydel (1983). La valeur relativement faible de la hauteur moyenne de $P$. erinaceus observée par Petit (2000) pourrait être liée à l'effet de la latitude sur la conformation de l'arbre. La forêt classée de l'Ouémé Supérieur est située entre les latitudes $9^{\circ} 11$ et $9^{\circ} 47$ alors que les formations naturelles à dominance de $P$. erinaceus du Burkina-Faso sont situées dans la zone soudanienne entre les latitudes $9^{\circ} 30$ et $13^{\circ}$. Or, selon Bonkoungou (1999), les arbres de $P$. erinaceus deviennent de plus en plus petits lorsque la latitude augmente. Néanmoins, le diamètre moyen de $P$. erinaceus obtenu dans la présente étude $(36,9 \mathrm{~cm})$ est semblable à celle notée par Petit (2000) qui est de $36 \mathrm{~cm}$.

En dehors des paramètres discutés ci-dessus, les autres paramètres dendrométriques ne présentent pas de différences significatives de moyenne en savane et en forêt. La surface terrière globale du peuplement ligneux, estimée à $10,8 \mathrm{~m}^{2} /$ ha, est inférieure à celle obtenue par Sagbo (2000) pour la même forêt classée $\left(15,73 \mathrm{~m}^{2} / \mathrm{ha}\right)$ et nettement inférieure à celle obtenue par Yayi (1998) dans la même formation naturelle (34 $\left.\mathrm{m}^{2} / \mathrm{ha}\right)$. Les arguments avancés cidessus pour expliquer les différences de densité peuvent être repris pour expliquer les différences de surface terrière observées entre les différentes études. Le caractère grégaire de $P$. erinaceus pourrait être à la base de cette dominance en grosseur. La distribution spatiale agrégative notée dans la présente étude confirme aussi la dominance de $P$. erinaceus, tant en forêt qu'en savane. Notons néanmoins que, selon Dajoz (1975), la répartition agrégative ou contagieuse s'explique aussi par l'hétérogénéité du substrat et le mode de dissémination des espèces du peuplement. La valeur obtenue pour l'indice de Blackman en forêt comme en savane est plus importante que celle obtenue pour les populations de Karité (Vitellaria paradoxa) $\left(I_{\mathrm{B}}=5\right.$; Gnanglè, 2005), les populations de Néré $\left(I_{B}=2,6 ;\right.$ Gbedji, 2003) de la région de Parakou au Bénin et de l'Iroko (Milicia excelsa) en forêt dense sèche du Bénin (Azonkponon, 2001). Le facteur d'écorce de $P$. erinaceus n'est pas influencé par le type de formation. Selon Husch et al. (1982), la proportion du volume d'écorce d'un billon de bois est égale à $\left(1-k^{2}\right)$. Cette proportion est donc égale à $20,8 \%$ pour les bois de $P$. erinaceus des zones soudanienne et soudano-guinéenne du Bénin. La comparaison des deux forêts classées (Ouémé Supérieur et Wari-Maro) révèle que les paramètres dendrométriques présentent des valeurs significativement similaires dans les deux forêts. On a noté qu'en savane, les espèces les plus retrouvées (densité supérieure à 10 tiges/ha) sont d'une part, Isoberlinia spp et d'autre part, Burkea africana. En forêt, les essences les plus représentées sont Isoberlinia spp. et Anogeissus leiocarpa.

\section{STRUCTURE DES PEUPLEMENTS DE $P$. erinaceus}

En ce qui concerne la répartition en diamètre des arbres, la forme en «i obtenues pour l'ensemble du peuplement est caractéristique de la nature inéquienne et multispécifique des arbres. Dans le cas de $P$. erinaceus, on note une distribution unimodale, en cloche, caractéristique des peuplements monospécifiques. En effet, selon Rondeux (1999), les distributions unimodales sont caractéristiques des peuplements équiennes ou monospécifiques et la forme de la courbe est révélatrice des évènements liés à la vie des peuplements. Néanmoins, un peuplement inéquienne soumis à diverses pressions peut également avoir l'allure unimodale notée dans l'étude.

\section{RELATION HAUTEUR-DIAMETRE}

La relation entre la hauteur et le diamètre de $P$. erinaceus, établie pour les formations de savane et de forêt est de type logarithmique avec de faibles coefficients de détermination, caractéristique de la diversité de forme de ces arbres en peuplement. Sagbo (2000) arrive à la même conclusion en établissant une relation de type parabolique avec un coefficient de détermination de $37,4 \%$ pour la même essence. Selon Rondeux (1999), la courbe parabolique est souvent caractéristique des peuplements équiennes, ce qui permet alors d'émettre l'hypothèse que les arbres de $P$. erinaceus sont d'âges rapprochés.

\section{CONCLUSION}

Cette étude a permis de décrire les principales caractéristiques des peuplements à dominance de $P$. erinaceus et constituent dès lors, une première source d'information utile pour 
l'aménagement des formations naturelles concernées. Par ailleurs, l'étude a aussi permis de conclure que dans les formations naturelles de la zone soudanienne, la dominance de $P$. erinaceus s'exprime par des valeurs relativement élevées du diamètre moyen des arbres et de la contribution spatiale de l'essence. En particulier, nous avons mis en évidence que ces peuplements offrent un potentiel ligneux en bois d'œuvre et de service dont l'aménagement permettrait de tirer de la valeur ajoutée par la vente de bois, tout en luttant contre la désertification.

\section{REMERCIEMENTS}

Les auteurs tiennent à remercier le Réseau Africain de Recherches Forestières (AFORNET) qui a financé cette étude.

\section{REFERENCES}

Anonyme. 1981. Développement des ressources forestières. Rapport, Représentation FAO Bénin, $48 \mathrm{p}$.

Anonyme. 2006. Le bois en chiffre. Production industrielle (hors série). Sessi France, $16 \mathrm{p}$.

Aubreville A. 1970. Flore du Cameroon : Légumineuses Cesalpinoideae. Muséum National d'histoire naturelle, Paris, 270 p.

Azonkponon N. 2001. Conservation in situ de l'Iroko (Milicia excelsa W. C. Berg) dans l'aire culturelle vodoun au Bénin. Mém. de DESS, Université d'Abomey-Calavi, Bénin, 107 p.

Bonkoungou, E. G. 1999. A quick guide to multipurpose trees from around the world. Arkansas, USA. [online], fact net. http:// foodsecurity.info/food-security.info/ Winrock\%20Archive/p_erinaceus.html. Consulté le 03 mars 2007.

Dajoz R. 1975. Précis d'Ecologie. Gauthiers Villars, $4^{e}$ éd., 503 p.

Gbedji E. K. Y. 2003. Caractérisation morphologique et structurale des parcs à néré (Parkia biglobosa J. Br.) au Bénin. Mém. Ingénieur Agronome, Université d'AbomeyCalavi, Bénin, $124 \mathrm{p}$.

Gnanglè P. C. 2005. Parcs à karité (Vitellaria paradoxa Gaertn) (Sapotaceae) au Bénin : Importance socio-culturelle, caractérisations morphologique, structurale et régénération naturelle. Mémoire de DESS, Université d'Abomey-Calavi (Bénin), 107 p.

Husch B., Miller C. and T. Beers. 1972. Forest mensuration. Ronald Press Company, New-York, 410 p.

Petit S. 2000. Environnement, conduite des troupeaux et usage de l'arbre chez les agropasteurs peuls de l'ouest burkinabé. Approche comparative et systématique de trois situations : Barani, Kourouma, Ouangolodougou. Thèse de Doctorat, Université d'Orléans, France, 522 p.

Philip M. S. 2002. Measuring trees and forests. Cabi publishing, $2^{\text {nd }}$ edition, London, UK, $310 \mathrm{p}$.

Rondeux J. 1999. La mesure des arbres et des peuplements forestiers. Presses agronomiques de Gembloux, Gembloux, France, $521 \mathrm{p}$.

Sagbo P. 2000. Etude des caractéristiques dendrométriques des peuplements naturels à dominance Isoberlinia spp. : Cas de la forêt classée de l'Ouémé Supérieur au nord du Bénin. Mém. Ingénieur Agronome, Université d'Abomey-Calavi, Bénin, $124 \mathrm{p}$.

Sinsin B., Eyog Matig O., Assogbadjo A. E., Gaoué O. G. and T. Sinadouwirou. 2004. Dendrometric characteristics as indicators of pressure of Afzelia africana $\mathrm{Sm}$. trees dynamics in different climatic zones of Benin. Biodiversity and Conservation 13 (8) : 1555 - 1570.

Sokpon N. 2000. Diversité des écosystèmes terrestres et forêts sacrées au Bénin. Projet Stratégie nationale biodiversité, Rapport MEHU, $59 \mathrm{p}$.

Tarp-Johansen M. J., Skovsgaard J. P., Madsen S. F., Johannsen V. K. and I. Skovgaard. 1997. Compatible stem taper and stem volume functions for oak (Quercus robur $L$ and Quercus petratea Matt Liebl) in Denmark. Annales des Sciences Forestières 54 : $577-595$.

von Maydell H. J. 1983. Arbres et arbustes du Sahel. Leurs caractéristiques et leurs utilisations. Eschnorn, GTZ, 531 p.

White F. 1983. The vegetation of Africa. UNESCO, Nat. Ress. Res. 20 : 341 - 356.

Yayi A. 1998. Contribution à l'aménagement de la forêt classée de l'Ouémé Supérieur au Nord du Bénin : structure et dynamique des différents groupements végétaux. Mém. Ingénieur Agronome, Université d'AbomeyCalavi, Bénin, 141 p. 